\title{
EVALUACIÓN DEL CONOCIMIENTO SOBRE LOS FACTORES DE RIESGO ASOCIADOS A DEFECTOS CONGÉNITOS EN MUJERES EN EDAD REPRODUCTIVA EN PANAMÁ
}

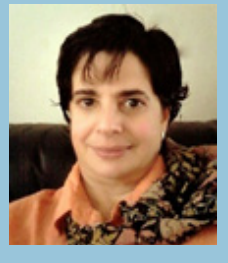

\section{Celia Llanusa Ruiz}

Universidad de Santander, Panamá

celia.1lanusa@usantander.edu.pa

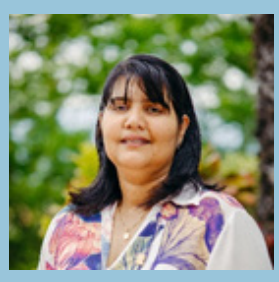

\section{Johana Gutiérrez Zehr}

Universidad de Santander, Colombia

coordinacion.investigacion@usantander.edu.pa

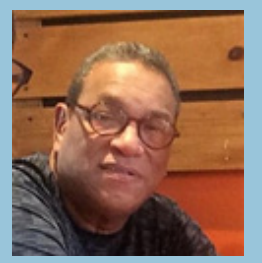

\section{Luis Sotillo Bent}

Complejo Hospitalario Arnulfo Arias Madrid.

Caja del Seguro Social, Panamá.

sotillo@cwpanama.net

\section{RESUMEN}

Las anomalías congénitas son la segunda causa de muerte en los niños menores de 28 días y de menos de 5 años en las Américas. Considerando los reportes de mortalidad en Panamá, el objetivo del estudio fue identificar el conocimiento sobre los factores de riesgo para los defectos congénitos en un grupo de mujeres en edad reproductiva en Panamá. Esta investigación fue de tipo descriptivo y prospectivo y se extendió de noviembre del 2015 a septiembre del 2016. La población estuvo integrada por todas las mujeres en edad reproductiva que acudieron a diversas instituciones vinculadas a los participantes del proyecto en ciudad Panamá, siendo el estimado 
de 1000 mujeres. La muestra estuvo formada por 400 mujeres, que representaron el 40\% de la población y que desearon voluntariamente participar en la investigación para la que se aplicaron cuestionarios. La muestra se calculó con un nivel de confianza del 99\% y un margen de error del 5\%. Los datos se tabularon de manera anónima y se procesaron mediante EPI-INFO, determinándose frecuencia y porcentajes, que permitieron hacer comparaciones. Al menos una tercera parte de las entrevistadas, no fue capaz de identificar cada factor de riesgo evaluado, de forma correcta. Dentro de los factores de riesgo relacionados con defectos congénitos, el uso de sustancias psicoactivas no fue reconocido igualmente por el mismo numero de entrevistadas. Resultó alarmante que más de la mitad de estas mujeres, no consideran que ingerir cualquier cantidad de alcohol en la gestación, es un factor de riesgo. Existe un deficiente conocimiento según la percepción por parte de las mujeres entrevistadas, de los factores involucrados en el origen de las anomalías congénitas, muchos de ellos controlables y cuya exposición puede evitarse en el embarazo. Esto lleva a reflexionar sobre la necesidad de desarrollar estrategias preventivas.

Palabras clave: Anomalías congénitas, defectos congénitos, embarazo, prevención.

\title{
EVALUATION OF KNOWLEDGE ON RISK FACTORS ASSOCIATED WITH CONGENITAL DEFECTS IN WOMEN IN REPRODUCTIVE AGE IN PANAMA
}

\begin{abstract}
Congenital anomalies are the second cause of death in children under 28 days and less than 5 years in the american region. Considering the mortality reports in Panama, the objective of the study was to identify the knowledge about the risk factors for congenital defects, in a group of women of reproductive age in Panama. This research was descriptive and prospective and extended from November 2015 to September 2016. The population was composed of all women of reproductive age who attended various institutions linked to the project participants in Panama City, being the estimated 1000 women. The sample consisted of 400 women, who represented $40 \%$ of the population and who voluntarily wished to participate in the research for which questionnaires were applied. The sample was calculated with a confidence level of $99 \%$ and a margin of error of 5\%. The data were tabulated anonymously and processed by EPI-INFO, determining frequency and percentages, which allowed making comparisons. At least one third of the interviewees were not
\end{abstract}


Evaluación del conocimietno sobre losfactores de riesgos asociados a defectos congénitos en mujeres en edad reproductiva en Panamá

Celia Llanusa Ruiz - Johana Gutiérrez Zehr - Luis Sotillo Bent (70:83)

able to identify each risk factor evaluated, correctly. Among the risk factors related to congenital defects, the use of psychoactive substances was not recognized equally by the same number of interviewees. It was alarming that more than half of these women, do not consider that drinking any amount of alcohol in pregnancy is a risk factor. There is a deficient knowledge according to the perception of the women interviewed, of the factors involved in the origin of the congenital anomalies, many of them controllable and whose exposure can be avoided in pregnancy. This leads to reflect on the need to develop preventive strategies.

Key words: Congenital anomalies, congenital defects, pregnancy, prevention.

\section{INTRODUCCIÓN}

Los defectos congénitos (DC), han pasado a constituir un problema de salud en los países desarrollados y en vías de desarrollo, al reducirse la mortalidad infantil por enfermedades infecciosas agudas (Situación de salud de Panamá, 2013). Las anomalías congénitas son la segunda causa de muerte en los niños menores de 28 días y de menos de 5 años en las Américas. En el mundo, afectan a 1 de cada 33 bebés y causan 3,2 millones de discapacidades. (Anomalías congénitas, 2015).

Los defectos congénitos graves más frecuentes son las malformaciones cardíacas, los defectos del tubo neural y el síndrome de Down. Estas anomalías congénitas pueden tener un origen genético, infeccioso o ambiental, ser de etiología multifactorial (Zomerdijk IM, 2015), aunque en muchos casos resulta difícil identificar su causa.

Es posible prevenir un grupo importante de estas anomalías. Hay medidas de prevención fundamentales como la vacunación, la ingesta suficiente de ácido fólico y yodo mediante el enriquecimiento de alimentos básicos, el suministro de complementos, los cuidados prenatales adecuados, evitar la exposición a ciertos medicamentos y drogas, entre otras.

Partiendo de este presupuesto, se decidió realizar esta investigación con el objetivo de identificar el conocimiento sobre los factores de riesgo para los defectos congénitos en un grupo de mujeres en edad reproductiva en Panamá. Con los elementos obtenidos se evaluarán estrategias y 
proyecciones con respecto a los servicios de genética y de la atención preconcepcional y prenatal, para encaminar esfuerzos a la prevención de defectos congénitos y a la reducción de la mortalidad infantil.

\section{METODOLOGÍA}

Esta investigación fue de tipo descriptivo de corte prospectivo. Se realizó en diversas instituciones de salud de ciudad de Panamá, con previa autorización para desarrollar el estudio. Se confeccionó la encuesta y fue discutida con el equipo de investigación y posteriormente validada. La investigación se inició en Noviembre del 2015 y culminó en septiembre del 2016.

La población estuvo conformada por el total de mujeres que acudieron a las instituciones vinculadas con el proyecto en el período señalado en la ciudad de Panamá, con un estimado de 1000 personas. La muestra estuvo representada por 400 mujeres que desearon participar voluntariamente en el diligenciamiento del cuestionario y que acudieron a dichas instituciones en el periodo de estudio (representando el $40 \%$ de la población). Esta muestra se calculó con un nivel de confianza del $99 \%$ y un margen de error del 5\%.

El instrumento se aplicó después de explicar a los participantes por parte del investigador o encuestador, las características del estudio y posterior a que ellas expresaran el deseo de colaborar con la investigación. En ningún momento se trabajó con el nombre de la mujer, ni otros datos de identificación personal, debe aclararse que la encuesta no lo requiere. Se mantuvo la ética profesional, confidencialidad de la información y se respetaron los principios de la ética médica. Se explicó que el objetivo era indagar el conocimiento que tiene una población femenina, acerca de los factores de riesgo reproductivos. Se utilizó el método de autoaplicación para el diligenciamiento del cuestionario, que contó con 19 preguntas.

La encuesta evaluó el nivel de conocimiento sobre los factores de riesgo asociados a defectos congénitos en mujeres en edad reproductiva e indagó sobre datos y antecedentes de la gestante (3 preguntas) y 19 preguntas que evaluaban conocimientos. Estas últimas se agruparon, discutiéndose 11 en el presente artículo. Inicialmente se realizó una evaluación de la encuesta en 15 gestantes y se decidió hacer modificaciones de la misma, en la redacción de dos preguntas (5 y 17). 
El cuestionario fue aplicado por los investigadores y colaboradores del estudio, permitiendo evaluar variables para caracterizar la muestra como: sociodemográficas(edad), historia reproductiva: gestación previa y antecedente de defecto congénito. Del resto de las variables se evaluó el conocimiento de su relación con el riesgo incrementado de defectos congénitos, seleccionándose para esta publicación: la edad materna, antecedente de hijo o familiares de ella o su esposo con defectos al nacimiento o enfermedad genética, abortos previos, historia de consanguinidad, uso de sustancias psicoactivas, exposición a radiaciones y a altas temperaturas.

El procesamiento de los datos se realizó de forma automatizada mediante el programa EPIINFO versión 7.2, donde también se creó la base de datos. Los resultados obtenidos se presentaron en tablas, las cuales permitieron su análisis.

\section{Resultados y discusión}

El análisis de las variables arrojó los resultados que se muestran a continuación. Cada variable fue analizada por separado. Seguidamente se evidencian los resultados relacionados con datos demográficos (edad), asi como con antecedentes patológicos personales.

La distribución de las encuestadas se realizó en tres grupos etarios como muestra la tabla 1, por debajo de 20 años son consideradas adolescentes y por encima de 35 años se considera avanzada edad materna. La mayoría de las mujeres se encontraban en el grupo de 20 a 34 años, que se considera edad fértil.

Tabla 1. Distribución de las encuestadas por edad.

\begin{tabular}{|c|c|c|}
\hline Edad & Frecuencia & Porcentaje \\
\hline$>20$ & 100 & $25 \%$ \\
\hline $20-34$ & 223 & $55.75 \%$ \\
\hline$>35$ & 77 & $19.25 \%$ \\
\hline Total & 400 & $100.0 \%$ \\
\hline
\end{tabular}

Fuente: Autores.

Se evalúo el porcentaje de encuestadas que habían estado embarazadas, considerando que 
esto podría modificar el conocimiento sobre los factores de riesgo y la prevención de defectos congénitos. El 45\% de estas mujeres refirió un embarazo previo, como se observa en la tabla 2. Resulta muy interesante el poco conocimiento que existe sobre los factores de riesgo en el embarazo y que se evidencia en la investigación, a pesar de que un alto porcentaje de estas mujeres tenía una gestación previa.

Tabla 2. Antecedentes de haber estado embarazada.

\begin{tabular}{|c|c|c|}
\hline Ha estado embarazada & Frecuencia & Porcentaje \\
\hline $\mathrm{Si}$ & 180 & $45.0 \%$ \\
\hline $\mathrm{No}$ & 220 & $55.0 \%$ \\
\hline Total & 400 & $100.0 \%$ \\
\hline
\end{tabular}

Fuente: Autores.

Para terminar de caracterizar la muestra y considerando los intereses del estudio, se indagó sobre el antecedente de un hijo con defecto congénito, pues el conocimiento adquirido en el embarazo anterior en relación con esta patología, podía modificar el resultado de la encuesta. Un bajo porcentaje de encuestadas ha tenido hijos con defectos congénitos (2.8\%). Estas fueron encuestadas fundamentalmente en los hospitales que ofrecen servicios de atención prenatal (ver tabla 3).

Tabla 3. Tiene algún hijo con defecto congénito.

\begin{tabular}{|c|c|c|}
\hline $\begin{array}{l}\text { Tiene algún hijo con defecto } \\
\text { congénito }\end{array}$ & Frecuencia & Porcentaje \\
\hline $\mathrm{Si}$ & 11 & $2.75 \%$ \\
\hline $\mathrm{No}$ & 389 & $97.25 \%$ \\
\hline Total & 400 & $100.0 \%$ \\
\hline
\end{tabular}

Fuente: Autores.

En la encuesta se realizaron un grupo de afirmaciones y la mujer debía marcar aquella que consideraba era factor de riesgo, para que el bebé de cualquier embarazada pudiera sufrir una anomalía durante su desarrollo o tener un defecto al nacer. La indagación sobre el conocimiento de los factores de riesgo, mostró los resultados que se discuten a continuación.

Los antecedentes familiares y personales positivos de defectos congénitos, malformaciones 
Evaluación del conocimietno sobre losfactores de riesgos asociados a defectos congénitos en mujeres en edad reproductiva en Panamá

Celia Llanusa Ruiz - Johana Gutiérrez Zehr - Luis Sotillo Bent (70:83)

fetales, enfermedades genéticas; incrementan el riesgo de recurrencia de anomalías al nacimiento. Estudios realizados en Europa y Estados Unidos han demostrado que entre el 25 y el $30 \%$ de todas las muertes después de las 28 semanas de gestación y durante las primeras semanas de vida, se deben a serias anomalías estructurales y en el $80 \%$ de los casos se han descrito factores genéticos y ambientales, con un riesgo del $1 \%$ o mayor de repetirse en otros embarazos (Taboada N, 2006).

En la presente investigación el $38 \%$ de las participantes no consideró de interés los antecedentes positivos familiares de defectos congénitos (ver tabla 4).

Tabla 4. Afirmación 1. Tiene un hijo anterior o familiares de ella o su esposo, con defectos al nacimiento o enfermedad genética.

\begin{tabular}{|c|c|c|}
\hline $\begin{array}{l}\text { Tiene un hijo anterior o familiares de ella o su } \\
\text { esposo, con defectos al nacimiento o enfermedad } \\
\text { genética }\end{array}$ & Frecuencia & Porcentaje \\
\hline $\mathrm{Si}$ & 247 & $61.75 \%$ \\
\hline $\mathrm{No}$ & 153 & $38.25 \%$ \\
\hline Total & 400 & $100.0 \%$ \\
\hline
\end{tabular}

Fuente: Autores.

Como se expresó anteriormente, se conoce que las edades reproductivas extremas, se relacionan con un incremento del riesgo para malformaciones congénitas. (Donoso, 2014).

El incremento de la edad materna, de los 35 años en adelante, se relaciona con un aumento del riesgo de defectos congénitos, en especial anomalías cromosómicas. En la población estudiada, el 35\% de la población no reconoció la edad materna avanzada como factor de riesgo. Por otra parte, el embarazo en la adolescencia, fue reconocido como factor de riesgo para tener hijos con anomalías congénitas y complicaciones perinatales solo por el $32 \%$ de la población, lo que constituye una preocupación y evidencia la necesidad de realizar labores de prevención primaria (ver tabla 5). 
Tabla 5. Afirmaciones 2 y 3. Tiene 35 años o más y tiene menos de 20 años.

\begin{tabular}{|c|c|c|}
\hline Tiene 35 años o más & Frecuencia & Porcentaje \\
\hline $\mathrm{Si}$ & 259 & $64.75 \%$ \\
\hline No & 141 & $35.25 \%$ \\
\hline Total & 400 & $100.0 \%$ \\
\hline Tiene menos de 20 años & Frecuencia & Porcentaje \\
\hline $\mathrm{Si}$ & 128 & $32.0 \%$ \\
\hline No & 272 & $68.0 \%$ \\
\hline Total & 400 & $100.0 \%$ \\
\hline
\end{tabular}

Fuente: Autores.

Diversos estudios muestran que el antecedente de abortos espontáneos ha mostrado asociación con las malformaciones congénitas. El estudio de Almaguer (2002), mostró asociación con un OR (Odd Ratio) muy significativo. La investigación mostró que el 45\% de las mujeres desconocían que el antecedente de abortos espontáneos es un factor de riesgo relacionado con las anomalías congénitas (ver tabla 6).

Tabla 6. Afirmación 4. Tuvo abortos anteriores ( 2 o más)

\begin{tabular}{|c|c|c|}
\hline Tuvo abortos anteriores (2 o más) & Frecuencia & Porcentaje \\
\hline $\mathrm{Si}$ & 218 & $54.5 \%$ \\
\hline No & 182 & $45.5 \%$ \\
\hline Total & 400 & $100.0 \%$ \\
\hline
\end{tabular}

Fuente: Autores.

Como se mencionó previamente, la consanguinidad juega un papel fundamental en la etiología de las enfermedades genéticas autosómico recesivas, anomalías congénitas y enfermedades multifactoriales. El riesgo es mayor mientras más cercano es el parentesco. Los matrimonios consanguíneos son frecuentes en comunidades aisladas y en determinados grupos étnicos (Arriaga, 2014). En Panamá existen zonas geográficas con alto índice de consanguinidad, por lo que resulta realmente importante conocer la influencia de esta en la aparición de anomalías congénitas. De las mujeres encuestadas, cerca del $40 \%$ no reconoció la consanguinidad como un factor de riesgo (ver tabla 7). 
Tabla 7. Afirmación 5. La gestante y su pareja, tienen alguna relación de parentesco (primos lejanos u otra).

\begin{tabular}{|c|c|c|}
\hline $\begin{array}{c}\text { La gestante y su pareja, tienen alguna } \\
\text { relación de parentesco (primos lejanos } \mathrm{u} \\
\text { otra) }\end{array}$ & Frecuencia & Porcentaje \\
\hline $\mathrm{Si}$ & 242 & $60.5 \%$ \\
\hline $\mathrm{No}$ & 158 & $39.5 \%$ \\
\hline Total & 400 & $100.0 \%$ \\
\hline
\end{tabular}

Fuente: Autores.

En la tabla 8, la afirmación 6 muestra que aproximadamente 1/3 de la población entrevistada, no considera que grandes cantidades de alcohol sean perjudiciales para la salud y casi la mitad, como puede verse en la misma tabla, considera que cualquier cantidad de alcohol (afirmación 7) puede ingerirse y no constituye factor de riesgo en el embarazo. El consumo de alcohol en el embarazo afecta el producto de la gestación. En 1957, la doctora Jacqueline Rouquette, pediatra francesa, describió por primera vez el síndrome alcohólico fetal (FAS, sigla en inglés de fetal alcohol syndrome) en su tesis doctoral (Evrard, 2010).

El alcohol en la sangre de la madre pasa de la placenta al embrión o feto a través del cordón umbilical, produciendo un número de alteraciones importantes en este (CDC, 2014). No se ha definido qué cantidad de alcohol es segura para que una mujer pueda consumir durante la gestación sin correr riesgo de daño fetal, por lo que se recomienda no ingerirlo. De igual forma no se ha definido si existe una etapa del embarazo considerada segura para tomar alcohol y no hay ningún tipo de bebida alcohólica que no represente riesgo.

El hábito de fumar se ha asociado con alteraciones embrio-fetales (Nazer, 2004). El tabaquismo se asocia a bajo peso al nacer, incrementa el riesgo de parto pretérmino, desprendimiento placentario, muerte fetal y abortos espontáneos, así como aumento del riesgo de infecciones del tracto respiratorio en el recién nacido. Estudios recientes han demostrado que el tabaquismo se encuentra dentro de los factores ambientales de tipo químico, asociado a cardiopatías congénitas (Blanco ME, 2009). No obstante, el 35\% de las encuestadas no consideró el hábito de fumar (afirmación 8) como factor de riesgo (ver tabla 8).

El consumo de drogas ilícitas por las gestantes, en especial de la cocaína, ha sido asociado a 
numerosas alteraciones congénitas como malformaciones génito-urinarias, alteraciones cardiacas, anomalías del Sistema Nervioso Central, SNC, alteraciones oftalmológicas o alteraciones en las extremidades (Donoso B, 2012). A pesar de lo que se insiste en todos los medios de los efectos perjudiciales de las drogas, aún no se reconoce por la población, el alcance del daño. La encuesta arrojó que el 34\% de la población, no considera el consumo de drogas (afirmación 9) como un factor de riesgo en el embarazo (ver tabla 8). Esto habla de la necesidad de realizar actividades de promoción de salud en la población, en especial con las mujeres en edad reproductiva.

Tabla 8. Resultados de la evaluación del uso de drogas psicoactivas en el embarazo.

\begin{tabular}{|c|c|c|}
\hline $\begin{array}{l}\text { Afirmación 6. Ingiere grandes cantidades de alcohol } \\
\text { (consumo medio regular de 40g diarios de alcohol. } \\
\text { Calcule que una bebida estándar contiene entre 12 y } \\
\text { 14 gramos de alcohol.) }\end{array}$ & Frecuencia & \\
\hline $\mathrm{Si}$ & 259 & $64.75 \%$ \\
\hline $\mathrm{No}$ & 141 & $35.25 \%$ \\
\hline Afirmación 7. Ingiere cualquier cantidad de alcohol & Frecuencia & Porcentaje \\
\hline $\mathrm{Si}$ & 194 & $48.5 \%$ \\
\hline No & 206 & $51.5 \%$ \\
\hline Afirmación 8. Tiene hábito de fumar & Frecuencia & Porcentaje \\
\hline Si & 257 & $64.25 \%$ \\
\hline No & 143 & $35.75 \%$ \\
\hline Afirmación 9. Consume drogas ilícitas & Frecuencia & Porcentaje \\
\hline So & 264 & $66.0 \%$ \\
\hline Total $*$ & 136 & $34.0 \%$ \\
\hline & 400 & $100.0 \%$ \\
\hline
\end{tabular}

Fuente: Autores.

*este es el total para cada una de las afirmaciones evaluadas.

La hipertermia al principio en el embarazo, especialmente en el primer trimestre, aumenta el riesgo de malformaciones congénitas como los defectos del tubo neural (DTN). Algunos estudios han encontrado un pequeño aumento en el riesgo de defectos cardiacos, defectos en la pared abdominal o hendidura oral cuando ocurre una fiebre a principios del embarazo, especialmente si es mantenida. (OTIS, 2015). El presente estudio mostró que el 58\% de las mujeres no consideran las altas temperaturas como factor de riesgo (ver tabla 9). 
Evaluación del conocimietno sobre losfactores de riesgos asociados a defectos congénitos en mujeres en edad reproductiva en Panamá

Celia Llanusa Ruiz - Johana Gutiérrez Zehr - Luis Sotillo Bent (70:83)

Tabla 9. Afirmación 10. Está expuesta a altas temperaturas (fiebre sostenida, trabaja en hornos con alta temperatura).

\begin{tabular}{|l|l|l|}
\hline $\begin{array}{l}\text { Está expuesta a altas temperaturas (fiebre sostenida, } \\
\text { trabaja en hornos con alta temperatura) }\end{array}$ & Frecuencia & Porcentaje \\
\hline $\mathrm{Si}$ & 168 & $42.0 \%$ \\
\hline No & 232 & $58.0 \%$ \\
\hline Total & 400 & $100.0 \%$ \\
\hline
\end{tabular}

Fuente: Autores.

El efecto de las radiaciones en el feto se ha reportado en estudios en animales, exposiciones humanas a la radiación diagnóstica y terapéutica, exposiciones a radiaciones de la bomba atómica en Hiroshima y Nagasaki en 1945 y a la explosión de la central nuclear de Chernóbil en abril de 1986 (Nazer, 2004). Estos estudios han reportado la relación de las radiaciones con malformaciones fetales, pérdida embrionaria precoz y efectos carcinogénicos en niños. Los riesgos de radiación durante el embarazo están relacionados con la etapa del embarazo y la dosis absorbida. Estos riesgos son mayores durante la organogénesis y en el período fetal temprano. (Shaw P, 2011). Las malformaciones que se presentan con mayor frecuencia son las del sistema nervioso central. La dosis requerida para producir efecto teratogénico de forma general, no supera la dosis que se recibe en exámenes diagnósticos (tomografías computarizadas de pelvis o exámenes de radiografía de diagnóstico convencionales), aunque estos niveles pueden alcanzarse con procedimientos intervencionistas fluoroscópicos de la pelvis y con radioterapia. El presente trabajo mostró (ver tabla 10), que el 33\% de las encuestadas no reconoció a las radiaciones (afirmación 11) como un factor de riesgo.

Tabla 10. Afirmación 11. Se expone a radiaciones (radiografías, trabajo con sustancias radioactivas, etc.).

\begin{tabular}{|l|l|l|}
\hline $\begin{array}{l}\text { Se expone a radiaciones (radiografías, trabajo con } \\
\text { sustancias radioactivas, etc.) }\end{array}$ & Frecuencia & Porcentaje \\
\hline $\mathrm{Si}$ & 266 & $66.5 \%$ \\
\hline No & 134 & $33.5 \%$ \\
\hline Total & 400 & $100.0 \%$ \\
\hline
\end{tabular}

Fuente: Autores. 
Durante la investigación se evidencia el escaso conocimiento de la población femenina entrevistada sobre los factores de riesgo. Esto constituye una preocupación especialmente considerando que todas estas son mujeres en edad reproductiva. Los resultados evidencian la necesidad de establecer estrategias de salud basadas en la promoción de salud, que permitan mejorar el conocimiento de los factores de riesgos para evitar o minimizar el riesgo de defectos congénitos.

\section{CONCLUSIONES}

El estudio ha mostrado el escaso conocimiento sobre los factores de riesgo para los defectos congénitos, en las mujeres entrevistadas, todas en edad reproductiva. Al menos 1/3 de las entrevistadas, no fue capaz de identificar cada factor de riesgo evaluado, de forma correcta.

Dentro de los factores de riesgo relacionados con defectos congénitos, el uso de sustancias psicoactivas no fue considerado por al menos una tercera parte de las entrevistadas. Resultó alarmante que más de la mitad de estas mujeres, no reconocen que ingerir cualquier cantidad de alcohol en la gestación, es un factor de riesgo.

Existe una pobre percepción por parte de las mujeres entrevistadas, de los factores involucrados en el origen de las anomalías congénitas, muchos de ellos controlables y cuya exposición puede evitarse en el embarazo. Esto lleva a reflexionar sobre la necesidad de desarrollar estrategias preventivas.

\section{RECOMENDACIONES}

Elaborar hojas informativas para los pacientes, donde se aborden los defectos congénitos más frecuentes y las recomendaciones para la prevención de las anomalías congénitas, con el fin de reducir el índice de malformaciones congénitas en el producto de la concepción de las gestantes afiliadas al sistema de salud de Panamá. 


\section{BIBLIOGRAFÍA}

ACOG. (30 de octubre de 2015). The American College of Obstetricians and Gynecologists. Obtenido de http://www.acog.org/Patients/Search-Patient-Education-Pamphlets-Spanish/Files/ La-reduccion-de-los-riesgos-de-defectos-congenitos

ALMAGUER P, F. M. (2002). Comportamiento de algunos factores de riesgo para malformaciones congénitas en la barriada de "San Lázaro". Rev Cubana Pediatr, 74(1), 4449. Recuperado el 19 de Diciembre de 2016, de http://scielo.sld.cu/scielo.php?script=sci arttext\&pid=S0034-75312002000100006\&lng=es\&nrm=iso

ANOMALÍAS CONGÉNITAS. (abril de 2015). Recuperado el 11 de noviembre de 2015, de OMS: http://www.who.int/mediacentre/factsheets/fs370/es/

ARRIAGA, M. (2014). Causas Endogenas geneticas. inasp, 1(12), 1-23.

BLANCO ME, A. S. (mayo-junio de 2009). Actualización sobre la cardiogénesis y epidemiología de las cardiopatías congénitas. Recuperado el 20 de noviembre de 2017, de http:// scielo.sld.cu/scielo.php?script=sci_arttext\&pid=S1684-18242009000300011

CDC. (2014). Guía para prevenir defectos de nacimiento. Obtenido de https://www.cdc.gov/ ncbddd/spanish/birthdefects/prevention.html

DONOSO B, O. E. (2012). Embarazo de alto riesgo. MEDWave, 12(5).

DONOSO, E. C. (2014). La edad de la mujer como factor de riesgo de mortalidad materna, fetal, neonatal e infantil168-174. Revista médica de Chile, 142(2), 168-174. Recuperado el 19 de noviembre de 2015, de http://www.scielo.cl/scielo.php?script=sci_arttext\&pid=S003498872014000200004\&lng=es\&tlng=es. 10.4067/S0034-98872014000200004.

EVRARD, S. (2010). Criterios diagnósticos del síndrome alcohólico fetal y los trastornos del espectro del alcoholismo fetal. Archivos argentinos de pediatría, 61-7.

NAZER, J. (Abril de 2004). Prevención primaria de los defectos congénitos. Rev. méd. Chile, 132(4), 501-508. Obtenido de http://www.scielo.cl/scielo.php?script=sci_arttext\&pid=S003498872004000400014\&lng=es. http://dx.doi.org/10.4067/S0034-98872004000400014

OTIS. (agosto de 2015). Recuperado el 2016 de diciembre de 16, de Motherbaby: https:// mothertobaby.org/es/fact-sheets/la-hipertermia/pdf/

SHAW P, D. A. (2011). Radiation exposure and pregnancy. Journal of vascular surgery, 53(1), 28S-34S.

SITUACIÓN DE SALUD DE PANAMÁ 2013. (2013). Obtenido de http://www.minsa.gob. 
Evaluación del conocimietno sobre losfactores de riesgos asociados a defectos congénitos en mujeres en edad reproductiva en Panamá Celia Llanusa Ruiz - Johana Gutiérrez Zehr - Luis Sotillo Bent (70:83)

$\mathrm{pa} / \mathrm{sites} / \mathrm{default} / \mathrm{files} /$ publicaciones/situacion_de_salud_panama_2013_0.pdf

TABOADA N, L. M. (2006). Comportamiento de algunos factores de riesgo para malformaciones congénitas mayores en el municipio de Ranchuelo. Rev Cubana Obstet Ginecol, 2, 1-13. Recuperado el 20 de Diciembre de 2016, de http://scielo.sld.cu/scielo.php?script=sci arttext\&pid=S0138-600X2006000200009\&lng=es\&nrm=iso

ZOMERDIJK IM, R. R. (2015). Dispensing of potentially teratogenic drugs before conception and during pregnancy: a population-based study. BJOG, 122(8), 1119-1129. 
Evaluación del conocimietno sobre losfactores de riesgos asociados a defectos congénitos en mujeres en edad reproductiva en Panamá

Celia Llanusa Ruiz - Johana Gutiérrez Zehr - Luis Sotillo Bent (70:83) 
Evaluación del conocimietno sobre losfactores de riesgos asociados a defectos congénitos en mujeres en edad reproductiva en Panamá Celia Llanusa Ruiz - Johana Gutiérrez Zehr - Luis Sotillo Bent (70:83) 